\title{
Large Optical Nonlinearity of Dielectric Nanocavity-Assisted Mie Resonances Strongly Coupled to an Epsilon-near-zero Mode
}

\author{
Kuidong Wang,${ }^{\dagger *}$ Ai-Yin Liu, ${ }^{\ddagger}$ Hui-Hsin Hsiao, ${ }^{\ddagger}$ Cyriaque Genet,${ }^{\dagger}$ Thomas Ebbesen ${ }^{\dagger *}$ \\ †University of Strasbourg, CNRS, ISIS \& icFRC, 8 allée Gaspard Monge, 67000 Strasbourg, France. \\ Institute of Electro-Optical Engineering, National Taiwan Normal University, Taipei 11677, Taiwan. \\ Email: kuidong.wang@unistra.fr, ebbesen@unistra.fr
}

\begin{abstract}
Strong coupling provides a powerful way to modify the nonlinear optical properties of materials.

The coupling strength of the state-of-the-art strongly coupled systems are restricted by weak field confinement of the cavity, which limits the enhancement of the optical nonlinearity. Here, we investigate a strong coupling between Mie resonant modes of high-index dielectric nanocavities and an epsilon-near-zero mode of an ultrathin indium tin oxide film and obtain an anti-crossing splitting of $220 \mathrm{meV}$. Static nonlinear optical measurements reveal a large enhancement in the intensity-independent effective optical nonlinear coefficients, reaching more than three orders of magnitude at the coupled resonance. In addition, we observe a transient response of $\sim 300$ fs for the coupled system. The ultrafast and large optical nonlinear coefficients presented here offer a new route towards strong coupling-assisted high-speed photonics.
\end{abstract}

KEYWORDS: strong coupling, dielectric nanocavity, Mie resonances, epsilon-near-zero mode, third-order optical nonlinearity 


\section{INTRODUCTION}

Strong coupling provides a new platform for manipulating the nonlinear optical properties of materials. In such systems, an optical mode is coupled resonantly to a given optical transition (optical mode, exciton or vibration), they both exchange energy faster than each dissipative and incoherent processes. ${ }^{1-4}$ Strong coupling can efficiently enhance many nonlinear optical phenomena of the coupled material, such as second-harmonic generation, ${ }^{5-7}$ third-harmonic generation, ${ }^{8,9}$ four-wave mixing ${ }^{10}$ as well as ultrafast nonlinearities. ${ }^{11,12}$ Apart from these specific nonlinear properties, the intrinsic third-order nonlinear response, which can be described by a nonlinear refractive index $n_{2}$ and a nonlinear absorption coefficient $\beta$, has also been studied very recently in the case of strong coupling, ${ }^{13}$ where strongly coupled J-aggregated molecules yielded two-orders-of-magnitude enhancement factors on both $n_{2}$ and $\beta$. These strong-coupling-enhanced nonlinear coefficients facilitate the high efficiency of various third-order nonlinear optical phenomena. However, the relative weak field confinement of the Fabry-Perot cavity used in previous work restricts the coupling strength, thereby limits the nonlinear enhancement. One possible option to enhance the local field is to use a plasmonic cavity, considering that a plasmonic structure can confine light into a subwavelength volume and thereby significantly boost the coupling strength. ${ }^{2}$ In a remarkable study, Boyd and coauthors showed that indeed very large thirdorder nonlinearities can be obtained when strongly couple the plasmonic antennas to an ultrathin indium tin oxide (ITO) film. ${ }^{14}$ Plasmonic nanostructures mainly enhance the electric near field, while affecting only weakly the magnetic near field. ${ }^{15}$ This diminishes the technological potential of such systems in the context of nonlinear optics, including light frequency conversion and optical modulation. ${ }^{16}$ 
High-index dielectric nanostructures appear promising as alternative candidates to provide optical modes required for strong coupling. Indeed, dielectric resonant nanocavities have various advantages, such as low optical loss, high radiation efficiency and reasonable bandwidth. Moreover, dielectric nanostructures with Mie-type resonances can sustain both electric dipolar (ED) and magnetic dipolar (MD) modes with comparable strength. ${ }^{15}$ In this case, the magnetic dipole stems from the circular displacement currents within each dielectric resonator induced by the incoming light. This makes it possible to enhance both the electric and magnetic near-fields at the nanoscale. ${ }^{15}, 17$ Considering their large breakdown threshold to laser power, dielectric nanostructures are expected to be an outstanding platform for various nonlinear optical phenomena, including harmonic generations ${ }^{18-23}$ and ultrafast optical switching. ${ }^{24-26}$

Taking advantage of the remarkable nonlinear optical properties of the high-index dielectric nanostructures and epsilon-near-zero (ENZ) media, ${ }^{27-29}$ here, we first present evidence of strong coupling between spectrally overlapping ED and MD modes of a dielectric nanocavity and an ENZ mode from an ultrathin ITO layer, reaching an anti-crossing splitting as large as 220 $\mathrm{meV}$. Then, by making use of the Z-scan technique, ${ }^{30}$ we observe large values of effective optical nonlinear coefficients of the system at the coupled resonance, that is more than three-orders-ofmagnitude larger than that of bare ITO and flat silicon films. These large optical nonlinearities result from the modified properties of the coupled system, which are related to the strong local field in these structures. In addition, the coupled system exhibits femtosecond response time of $\sim 300$ fs that stems from the thermalization of pump-induced hot electrons in both constituents. The large, ultrafast nonlinear response of this system is a very promising path for nonlinear photonics. 


\section{RESULTS AND DISCUSSION}

Linear optical measurements. The coupled system in our study consists of an amorphous silicon disk array, which was fabricated on top of a 33-nm ultrathin ITO layer, as shown in Figure 1a. The silicon disks, with an average height of $200 \mathrm{~nm}$, were fabricated on a sputtered silicon film with a standard electron-beam lithography (EBL) and a reactive ion etching (RIE) techniques (Figure 1b). The dimensions of the unit cell were $1 \mu \mathrm{m} \times 1 \mu \mathrm{m}$ in order to ensure strong far-field signal in transmittance measurements. The details of the sample's preparation are

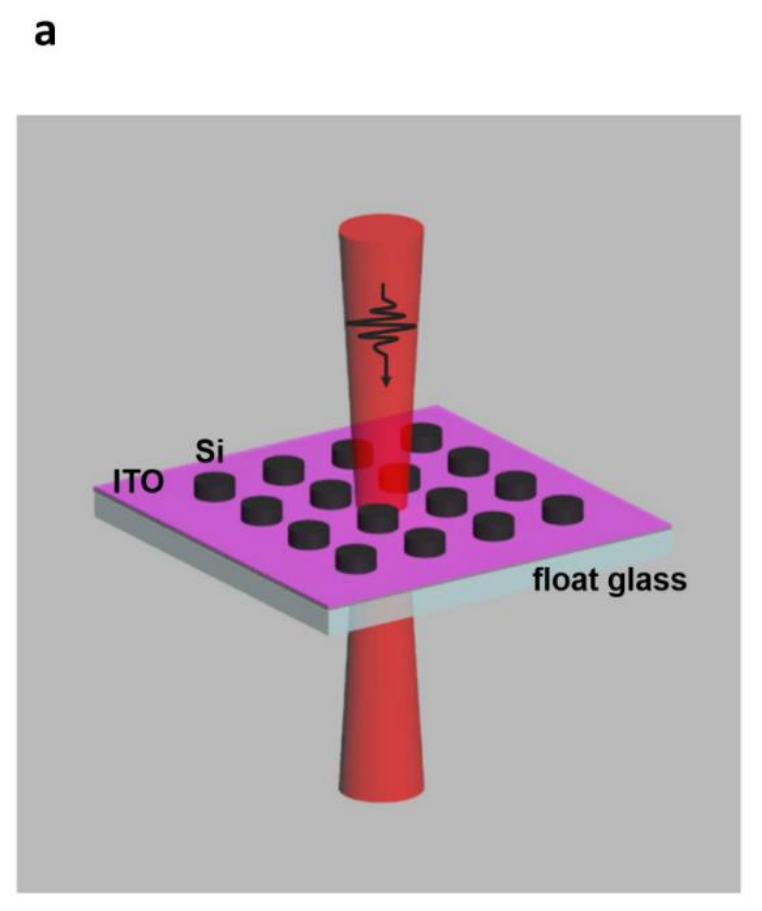

b

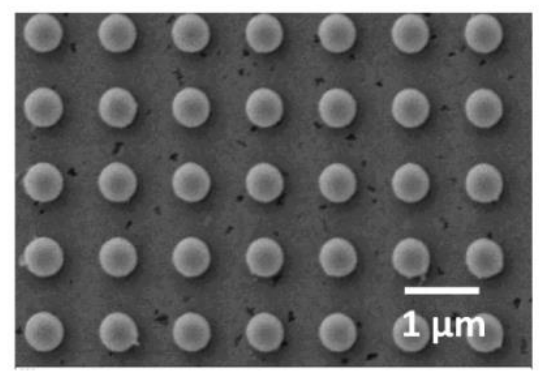

C

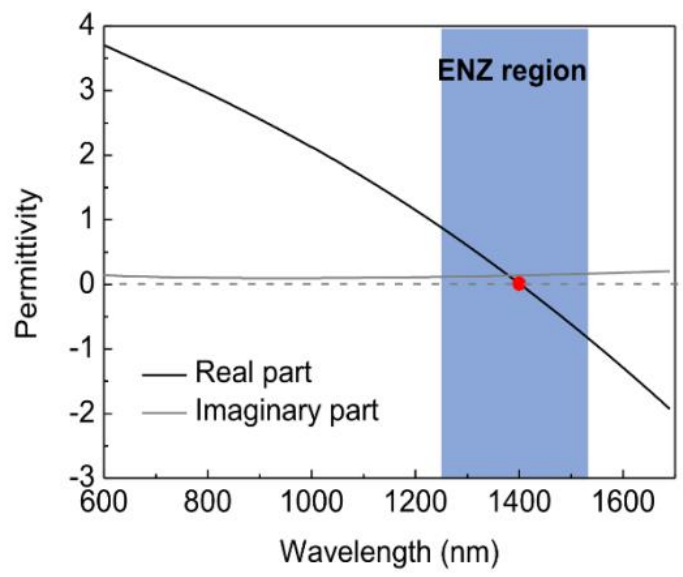

Figure 1. Schematic of the coupled system and permittivity of the ITO. (a) Illustration of the coupled system between silicon disks and ITO film. (b) SEM image of the silicon disks on top of an ITO layer. (c) Measured real (black curve) and imaginary (gray curve) parts of the permittivity of the ITO film used in this work. The ENZ region and ENZ wavelength are indicated by a blue shadow and a red dot, respectively. 
presented in the Methods part. In order to systematically explore the strong coupling between the $\mathrm{ED} / \mathrm{MD}$ resonant modes and the ENZ mode, the diameter of the silicon disk was intentionally designed to support localized ED/MD modes at the wavelengths corresponding to the ENZ region of the ITO (Figure 1c). The linear permittivity of the ITO layer was measured by a spectroscopic ellipsometry (M-2000, J.A. Woollam Co. Inc.), and the corresponding zero permittivity (real part) occurs at $1400 \mathrm{~nm}$, as presented in Figure 1c.

In order to explore the linear optical properties of the strongly coupled ENZ mode with the Mie resonances, it is necessary to briefly discuss the optical behavior of each constituent. The ENZ mode is a confined optical mode and can only be supported by an ultrathin layer of ENZ materials. ${ }^{31-33}$ Its dispersion relation can be derived by solving Maxwell's equations of a threelayer system, as follows ${ }^{33}$

$$
1+\frac{\varepsilon_{0} k_{z 2}}{\varepsilon_{2} k_{z 0}}=i \tan \left(k_{z 1} d\right)\left(\frac{\varepsilon_{1} k_{z 2}}{\varepsilon_{2} k_{z 1}}+\frac{\varepsilon_{0} k_{z 1}}{\varepsilon_{1} k_{z 0}}\right)
$$

where $k_{z i}^{2}=\varepsilon_{i} \frac{\omega^{2}}{c^{2}}-k_{\|}^{2}$ and $\varepsilon_{i}$ are the longitudinal wave number and the relative permittivity for free space $(i=0)$, ENZ material $(i=1)$ and glass substrate $(i=2)$, respectively. $k_{\|}$is the transverse wave number, $\omega$ is the angular frequency, $d$ is the film thickness of the ENZ material, and $c$ is the speed of light in free space. When the film thickness is much thinner than the skin depth of the ENZ material, such as the 33-nm ITO used in our study, the dispersion of the ENZ mode will be close to the ENZ frequency and stay nearly constant at small $k_{\|}$region, as shown in Figure 2a. Since the dispersion of the ENZ mode lies on the right side of the air light line, a hybrid structure that can support a wide range of wavevector components is required to efficiently excite the ENZ mode. ${ }^{34}$ In addition, the electric field intensity distribution of the ENZ mode can be calculated by using field-continuity conditions (inset of Figure 2a). Obviously, the field is concentrated inside 
the film, which enables high coupling efficiency when the ENZ material interacts with adjacent nanostructures.

Regarding the high-index dielectric nanostructure, a disk shape was used here because it can bring the lowest-order electric and magnetic dipolar resonances into spectral overlap within the ENZ region by choosing a suitable height-to-diameter aspect ratio. ${ }^{35}$ Therefore, it is sufficient to change the diameter of the disk while keeping the height and unit cell dimensions fixed in our simulations (COMSOL Multiphysics). The calculated transmittance spectra of the silicon disk array on a glass substrate (no ITO layer) are presented in Figure 2b. The overlapping ED and MD modes redshift as the diameter of the silicon disk increases, and eventually spectrally split when the disk diameter is larger than $\sim 570 \mathrm{~nm}$. This spectral separation takes place because the electric dipole resonance exhibits a much stronger redshift with respect to the nanodisk diameter as compared to the spectral shift of the magnetic dipole resonance based on the magnetic wall boundary condition of the cylindrical dielectric cavity. ${ }^{36}$ The result agrees well with the diameterdependent transmittance spectra of the silicon disks that discussed in the literature. ${ }^{35}$ The parameters of the silicon disks are intentionally selected here so that both the ED and MD modes can appear at the ENZ region of the ITO. 

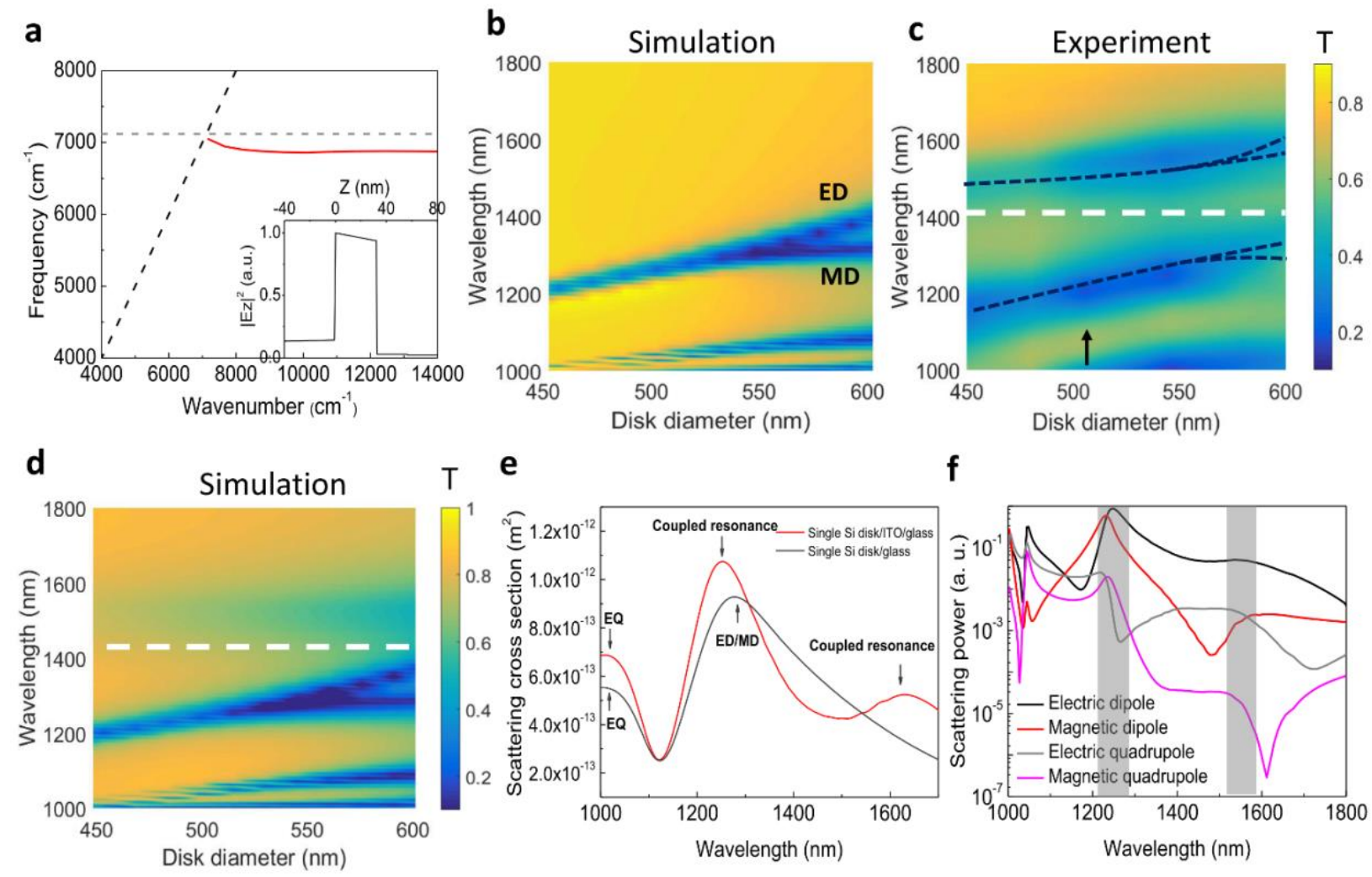

Figure 2. Linear optical responses of the coupled systems. (a) The dispersion relation of the ENZ mode (red curve) in air/33-nm ITO/glass system calculated from Eq. (1). The black dashed line is the air light line $\omega=c k_{\|}$and the gray dashed line denotes the ENZ frequency. Inset of (a), normalized electric field intensity distribution of the 33-nm ITO/substrate system. (b) Simulated transmittance spectra of silicon disks (without ITO) with respect to disk diameter. ED and MD stand for electric dipole and magnetic dipole, respectively. (c) and (d) are the measured and the simulated transmittance spectra of silicon disk-ITO coupled systems as a function of disk diameter, respectively. The white dashed line represents the ENZ mode and the black curves follow the peaks of the coupled resonances. The measured transmittance spectra at different silicon diameters are shown in Figure S1 in Supporting Information. Here the step size in the simulations of the disk diameter is $15 \mathrm{~nm}$. (e) Calculated scattering cross section of a single silicon disk (505-nm disk diameter) with (red curve) and without ITO layer (gray curve). (f) Multipolar decomposition of the 505-nm Si disk/ITO strongly coupled system with respect to wavelength. Gray areas correspond to the two strongly coupled hybrid resonances. 
The linear optical response of the silicon disk-ITO strongly coupled systems were recorded at normal incidence (Model V-670, JASCO). As shown in Figure 2c, the measured transmittance spectra of the coupled systems at various disk diameters exhibit pronounced splittings at $1400 \mathrm{~nm}$, as well as an anti-crossing feature. This feature is the signature of the strong coupling between the ENZ mode and nanodisk resonant modes. ${ }^{2,}{ }^{34}$ When the diameter of the silicon disk increases to $580 \mathrm{~nm}$, the splitting of the ED and MD components at both coupled resonances occur, which are consistent with the separation of two modes at large disk diameters in pure silicon disk as given in Figure $2 \mathrm{~b}$ (details are shown in Supporting Information S1). Interestingly, there are also resonances at $\sim 1000 \mathrm{~nm}$, that possibly come from the electric quadrupole (EQ) and collective resonance as discussed in Supporting Information S2. Similar characteristics are also displayed in the simulated spectra of the coupled systems (Figure 2d). In contrast to the wavelength position of the resonances, the linewidths and amplitudes of the measured spectra are different from that of the simulation. These differences mainly come from the imperfect disk shape and height unavoidable through the fabrication process, because the simulated resonances are sensitive to the shape and height of the silicon disk when the disk diameter is fixed. Another possible reason is the different between refractive index of each material used in our simulations and actual values in experiment. The strong coupling can also be proved by comparing the scattering cross section (SCS) spectrum of a single silicon disk with and without ITO layers (Figure 2e), here only localized resonances exist and there is no collective mode from grating effects. Considering a single Si disk/substrate system with 505-nm diameter disk, the overlapping ED/MD modes appear at $1290 \mathrm{~nm}$, which are close to ENZ wavelength. When an ultrathin ITO layer is inserted between the single silicon disk and the substrate, two new coupled resonances (one red branch and one blue branch with respect to the ENZ wavelength) are induced due to strong coupling. The coupled resonances in the SCS spectrum 
resemble that in measured transmittance spectrum (blue curve in Figure S1). Multipolar decomposition analysis indicates that the electric and magnetic dipoles dominate both the coupled resonances (Figure 2f). Although the electric quadrupole and magnetic quadrupole also play a role in the coupled resonances, their contribution can be neglected in our discussion due to their small amplitudes. This suggests that both the ED and MD modes are strongly coupled to the ENZ mode.

Nonlinear optical measurements. The remarkable optical nonlinear response of the ENZ material and high-index dielectric nanostructures lead us to examine the third-order optical nonlinearity of the strongly coupled system via Z-scan measurements. The details of our Z-scan setup was presented in Supplementary Information of our previous work. ${ }^{13}$. Usually, the thirdorder optical nonlinear process can be shown by a linear relation between the peak and valley contrast of the normalized transmittance $\left(\Delta T_{\mathrm{p}-\mathrm{v}}\right)$ and the peak intensity of the incident light at the focus. ${ }^{30}$ In our strongly coupled system, $\Delta T_{\mathrm{p}-\mathrm{v}}$ increases nearly linearly with the peak intensity at a representative wavelength (Supporting Information S3). This indicates that the transmittance variation in Z-scan measurements can be used to characterize the nonlinear refractive index and nonlinear absorption coefficient because of its third-order nonlinear origin. Here, the effective nonlinear coefficients, $\beta_{\text {eff }}$ and $n_{2 \text { eff }}$ are used to describe the third-order optical nonlinearity in silicon disk/ITO strongly coupled system. This way, we can estimate $\beta_{\text {eff }}$ and $n_{2 \text { eff }}$ from the traces of the open- and closed-aperture Z-scan, respectively (Supporting Information S4). Since both $\beta_{\text {eff }}$ and $n_{2 \text { eff }}$ are strongly dependent on wavelength (Figures $3 \mathrm{a}$ and $3 \mathrm{~b}$ ), the peak intensity required for measuring a transmittance change at wavelengths far from resonances will easily cause saturation for the wavelengths close to the resonances. To avoid this issue, lower peak intensities were used when measuring optical nonlinearity near the coupled resonances. The corresponding 
parameters of the laser used for the measurements at various wavelengths are given in Supporting Information S5.
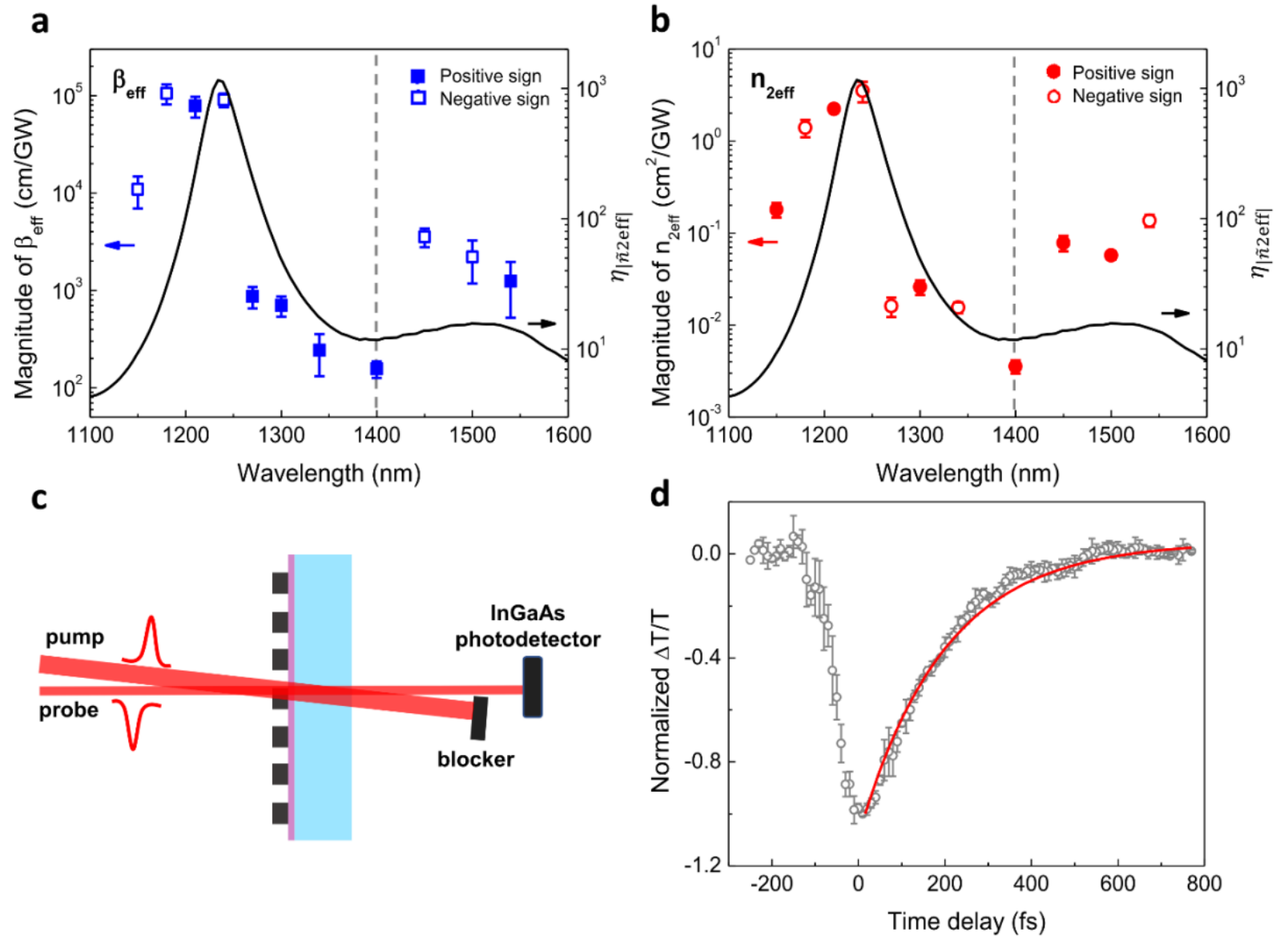

Figure 3. Nonlinear optical responses of the strongly coupled system. Magnitude of (a) $\beta_{\text {eff }}$ and (b) $n_{2 \text { eff }}$ for the strongly coupled system with 505-nm Si disk on an ITO film at various wavelengths. The positive and negative signs of the value are denoted as closed and open symbols, respectively. The black curves are the calculated dispersive enhancement factor of the effective complex nonlinear refractive index for the coupled system. The dashed gray lines denote ENZ wavelength. (c) Schematic of the pump-probe experimental setup. (d) Normalized ultrafast transmittance change of the silicon disk/ITO coupled system under an 1240-nm light pulse illumination. The red curve is the fitting curve for the decay process. The decay time is $200 \mathrm{fs}$. The pump fluence applied here is $110 \mu \mathrm{J} / \mathrm{cm}^{2}$. 
In order to explore the nonlinear response under optical strong coupling conditions, we choose a silicon disk/ITO coupled sample with the largest anti-crossing splitting (220 meV), which corresponds to a disk diameter of $505 \mathrm{~nm}$ (blue curve in Figure S1). As shown with the log plots in Figures $3 \mathrm{a}$ and $3 \mathrm{~b}$, both $\beta_{\text {eff }}$ and $n_{2 \text { eff }}$ display very large absolute values at the coupled resonances. By contrast, they exhibit relatively small values near ITO's ENZ wavelength. The trends of the dispersive nonlinear coefficients follow the linear transmittance spectrum of the coupled system, and the spectral positions of the maximum nonlinear coefficients match that of the coupled hybrid resonances (blue curve in Figure S1). More precisely, near the wavelength of one of the coupled resonances $(1240 \mathrm{~nm}), \beta_{\text {eff }}$ and $n_{\text {2eff }}$ reach the maximum absolute values of $9.41 \times 10^{4} \mathrm{~cm} / \mathrm{GW}$ and $3.51 \mathrm{~cm}^{2} / \mathrm{GW}$, which are more than three orders of magnitude larger than both the $\beta$ of a $200-\mathrm{nm}$ thick silicon film $(17.8 \mathrm{~cm} / \mathrm{GW})$ and the maximal $n_{2}$ of the bare $33-$ $\mathrm{nm}$ ITO at its ENZ wavelength $\left(1.71 \times 10^{-3} \mathrm{~cm}^{2} / \mathrm{GW}\right)$. At the wavelength of another coupled resonance $(\sim 1550 \mathrm{~nm})$, the effective nonlinearity coefficients are also enhanced by $\sim 2$ orders of magnitude compared with those of the bare ITO and flat silicon films. Because the optical nonlinearity of the ITO layer at ENZ wavelength is very small compared with that of the coupled system, it is important to explore the role of the ED/MD modes of the silicon disk on this optical nonlinearity enhancement. We investigated the open- and closed-aperture Z-scan measurements of a reference sample where a silicon-disk array was directly fabricated on a float glass substrate (no ITO layer). The Si disk/substrate sample has overlapping ED/MD resonances ( $1240 \mathrm{~nm}$, Fig. S6a) that are quite close to one of the coupled resonances in the Si disk/ITO/substrate system used in our optical nonlinearity measurements (blue curve in Figure S1). The values of $\beta$ and $n_{2}$ of the Si-disk/substrate at the ED/MD resonant wavelength are measured to be $9.46 \times 10^{3} \mathrm{~cm} / \mathrm{GW}$ and $0.42 \mathrm{~cm}^{2} / \mathrm{GW}$, respectively, which are still much smaller than that of the Si disk/ITO/substrate 
system at the coupled resonance (see Figure S6). This confirms that the strong-coupling-assisted new mode plays a major role on the large nonlinearity reported here. The Z-scan results of the bare ITO and the flat silicon films, as well as the Si-disk array/substrate (without ITO) are discussed in Supporting Information S6. In addition, the optical nonlinear coefficients at the coupled resonances measured here are also more than two orders of magnitude larger than that of reported plasmonic metamaterial, ${ }^{37}$ and more than one order of magnitude larger than that of the nonlinear ITO in the ENZ region ${ }^{38}$ and than that recently reported J-aggregated molecule in the strong coupling regime. ${ }^{13}$ The signs of the measured values of $\beta_{\text {eff }}$ and $n_{2 \text { eff }}$ with respect to the optical wavelength presented in Figures $3 \mathrm{a}$ and $3 \mathrm{~b}$ are important, the negative and positive values of $\beta_{\text {eff }}$ correspond respectively to the saturable absorption and reverse saturable absorption, while the positive and negative values of $n_{2 \text { eff }}$ denote the positive and negative "Kerr lens", respectively.

In contrast to the static optical nonlinear measurements recorded by the Z-scan technique, the time-resolved dynamics of the strongly coupled system was also exploited by modulating the amplitude of the transmitted probe light via a control beam. The schematic of the measurements is presented in Figure 3c, and the details of the pump-probe setup can be found in the Methods part. As shown in Figure 3d, with the illumination of the pump pulse, the transmittance of the probe light first reduces drastically within 100 fs near zero delay, which is restricted by the pulse width of the laser. The transmittance then recovers back to the original state within an $\sim 200$-fs exponential decay. The negative value of the normalized transmittance change corresponds to a light-induced absorption process for the coupled system, and the femtosecond Kerr-type nonlinearity results from the laser-induced thermalization of the hot electrons inside both the silicon disk and ITO film. ${ }^{24,38,39}$ The 300-fs switching time achieved here, which corresponds to a calculated bandwidth of $1.46 \mathrm{THz}$ for Gaussian pulses with a time-bandwidth product of $0.44,{ }^{40}$ 
is even shorter than the optical modulation in plasmonic antenna/ITO coupled system ${ }^{28}$ that we recently reported, making our silicon disk/ITO coupled system ideal for ultrafast optical processing and switching applications.

As we stressed above, we attribute the large nonlinear coefficients at the coupled resonances to the strong electromagnetic field enhancement of the silicon disk/ITO strongly coupled system. In order to correlate the optical nonlinear coefficients and the local field intensity $\left(I_{\text {loc }}\right)$, we analyze the electric field distribution at the coupled resonances by describing the electric field inside both the dielectric nanodisk and the ITO layer as a linear superposition of electric and magnetic components. ${ }^{41,42}$ Here we define an enhancement factor for the local electric field intensity as $\eta_{I}=I_{\text {loc }} / I_{0}$, where $I_{0}$ is the intensity of the incident light in vacuum. As we already explained in our former work, ${ }^{13,43}$ the enhancement of the effective third-order susceptibility $\left(\chi_{\text {eff }}^{(3)}\right)$ and $\eta_{I}$ can be directly related by a mean field approximation model within different nanocavity
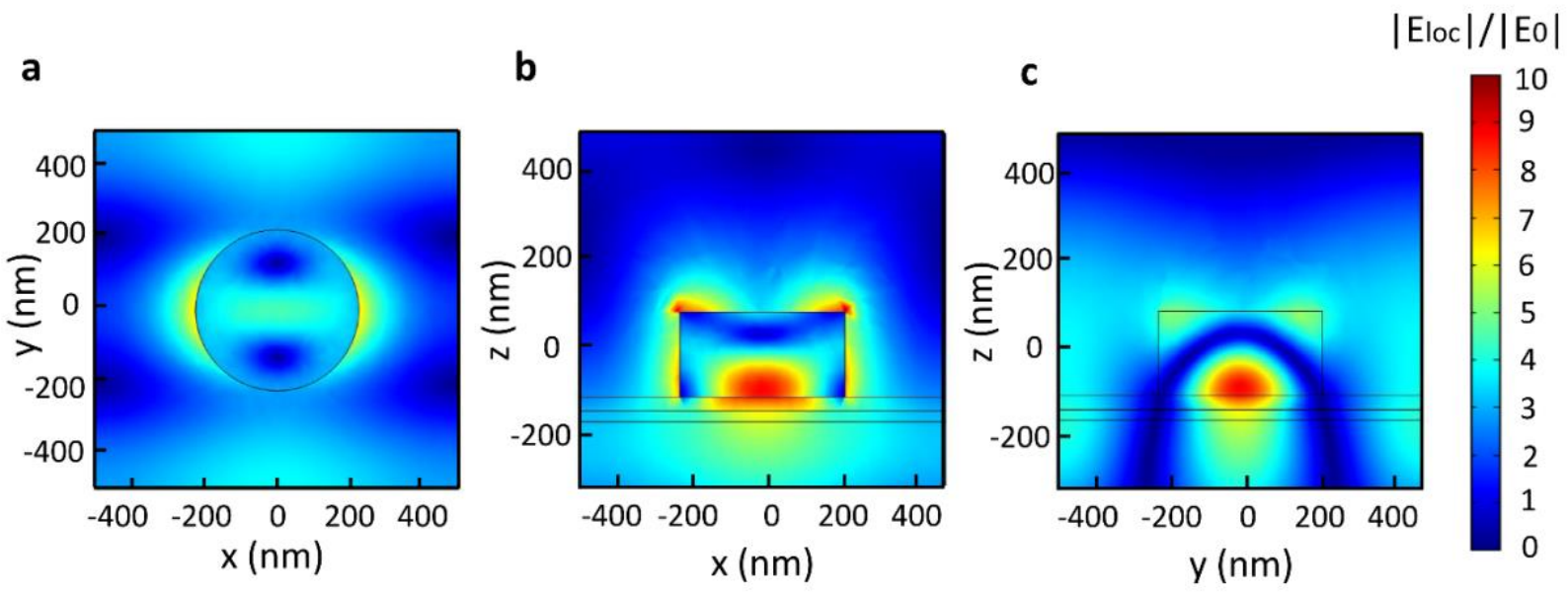

Figure 4. Electric field distribution of the Si disk/ITO system simulated at the coupled resonant wavelength of $1240 \mathrm{~nm}$. (a), (b) and (c) represent the electric field distributions at different crosssectional views of the silicon disk/ITO coupled system when the disk diameter is fixed to $505 \mathrm{~nm}$. Here the polarization direction of the normal incident light is parallel to the $x$ axis. 
systems. This approach leads to an enhancement factor of $\chi_{\text {eff }}^{(3)}$ that follows $\eta_{\chi(3) \text { eff }}=\eta_{I}^{2}$. Considering the local electric field distribution $\left(\mathrm{E}_{\mathrm{loc}}\right)$ at one of the coupled resonances $(1240 \mathrm{~nm})$ as presented in Figure $4, \eta_{I}$ is estimated to be $\sim 34$ by averaging an unit volume of both silicon disk and ITO layer based on the simulated electric field profile. This gives rise to a three-orders-ofmagnitude enhancement on $\chi_{\mathrm{eff}}^{(3)}$ at the resonant condition. A comparison with the electric field distribution at off-resonant wavelength $(1120 \mathrm{~nm})$ can also be found in Figure S7 in Supporting Information. Regarding to the optical Kerr effect induced by a single light beam, the intensityindependent effective complex nonlinear refractive index $\tilde{n}_{2 \text { eff }}$ follows ${ }^{44,45}$

$$
\tilde{n}_{2 \mathrm{eff}}=\frac{3}{4 n_{0 \mathrm{eff}} n_{0 \mathrm{eff}}^{\prime} \varepsilon_{0} c} \chi_{\mathrm{eff}}^{(3)}
$$

where $\tilde{n}_{2 \mathrm{eff}}=n_{2 \mathrm{eff}}+i n_{2 \mathrm{eff}}^{\prime \prime}=n_{2 \mathrm{eff}}+i \frac{c}{2 \omega} \beta_{\mathrm{eff}}, n_{0 \mathrm{eff}}$ and $n_{0 \mathrm{eff}}^{\prime}$ are the complex and the real part of the effective linear refractive index, respectively. $n_{2 \text { eff }}^{\prime \prime}$ is the imaginary part of the effective nonlinear refractive index. The relation clearly suggests that the effective nonlinear refractive index can be boosted in the same proportion as the enhancement of $\chi_{\mathrm{eff}}^{(3)}$. Therefore, by simulating the electric field intensity enhancement at various wavelengths (See Figure S8), the dispersive $\eta_{\chi(3) \text { eff }}$ and the wavelength-dependent enhancement of $\tilde{n}_{2 \text { eff }}\left(\eta_{\mid \tilde{n} 2 \text { eff } \mid}\right)$ can be estimated. Both are plotted as black curves in Figures $3 \mathrm{a}$ and $3 \mathrm{~b}$. As expected, the simulated enhancement of the effective nonlinear refractive index well follows the dispersive trend of the effective optical nonlinear coefficients of the strongly coupled system. It should be noted that equation (2) can be applied here because in our system the coupled resonant wavelengths are relatively far from the ENZ wavelength $(1400 \mathrm{~nm}) .{ }^{46,47}$ When considering an ENZ-effect dominated system, for instance a thick ITO film, the free carrier nonlinearity should also be considered, as discussed in detail in the literature. ${ }^{48-53}$ 
Although the large optical nonlinear coefficients of the silicon disk/ITO strongly coupled system are estimated from the data acquired under strong laser irradiance, the enhancement is independent on peak power of the incident light (Figure S3d), i.e. it is an intrinsic optical property of the coupled system.

\section{CONCLUSION}

We have observed the signature of optical strong coupling between the ED/MD modes of the silicon nanodisk and the ENZ mode of an ultrathin ITO layer. Through wavelength-dependent Z-scan measurements performed on this coupled system, we obtain large values on both $n_{\text {2eff }}$ $\left(3.51 \mathrm{~cm}^{2} / \mathrm{GW}\right)$ and $\beta_{\text {eff }}\left(9.41 \times 10^{4} \mathrm{~cm} / \mathrm{GW}\right)$ at the coupled resonant wavelength. These large nonlinearities are ascribed to a significant enhancement of the electric field in the coupled system. In addition, the coupled system demonstrates an ultrafast modulation of its optical response of $\sim 300$ fs.

Since the optical nonlinearity coefficients of the coupled system are strongly related to the local electric field enhancement, designing a specific dielectric nanocavity with higher field confinement could be a direct way to further improve the values of $n_{2 \text { eff }}$ and $\beta_{\text {eff }}$ for the system. Because the excitons of semiconductors also show remarkable optical nonlinearities, ${ }^{54}$ one can expect large optical nonlinearity by coupling the excitons to the ED/MD modes of the dielectric nanocavity. The study of ultrafast, large third-order optical nonlinearity in a dielectric nanodisk/ITO coupled system here offers a new route to explore various third-order optical nonlinear phenomena via strong coupling for high speed active photonic and optoelectronic devices. 


\section{METHODS}

Sample preparation. The sample used in our study is a silicon nano-disk array which was fabricated on top of a commercially 33 -nm ITO layer (sheet resistance $50 \Omega$ /square) via standard EBL and RIE techniques. Between the ITO film and the substrate, there is an $\sim 25-\mathrm{nm} \mathrm{SiO}_{2}$ passivation layer in order to ensure electric insulation. Before fabrication, an 200-nm thick amorphous silicon film was sputtered on top of the ITO layer $(0.21 \mathrm{~nm} / \mathrm{s}$, PLASSYS MP300). For the EBL process, two layers of the positive PMMA e-beam resists (system 600K/950K of AR-P series, AllresistGmbH) were used in order to improve the accuracy of the exposed pattern. The 600-K and 950-K resists were spin coated sequentially with r.p.m. of 4000 and 2000, respectively, on top of the silicon film. The film thickness of the $600-\mathrm{K}$ and $950-\mathrm{K}$ resists are 220 and $100 \mathrm{~nm}$, respectively. After baking the spin coated resists at $180^{\circ} \mathrm{C}$ for $90 \mathrm{~s}$, the designed areas were exposed to a 30-keV electron beam (AURIGA, Zeiss). The exposed part of resist was then developed in a conventional solution of methyl isobutyl ketone-isopropyl alcohol (MIBK-IPA) (1:3) for $30 \mathrm{~s}$ and then rinsed with an IPA solution for $30 \mathrm{~s}$ to complete the development process. Next, the Cr mask was obtained by evaporating a 30-nm chrome (Cr) film $(0.05 \mathrm{~nm} / \mathrm{s}$, PLASSYS ME300) and removing the unexposed resist with acetone (lift-off process). The Cr disks can then be transferred to the silicon film by using $\mathrm{Ar}$ and $\mathrm{CHF}_{3}$ plasma gases under the pressure of $2 \times 10^{-5}$ Torr in a RIE chamber (PlasmaLab 80 Plus, Oxford Inst.). Finally, the Cr mask was removed by immersing the sample in a Chromium etchant solution in $\sim 2$ minutes. The silicon disks, which possess average diameters from $450 \mathrm{~nm}$ to $600 \mathrm{~nm}$, were designed so that they support localized ED and MD modes at wavelengths that can strongly couple to the ENZ mode of ITO at $1400 \mathrm{~nm}$. The choice of the unit cell dimensions is also crucial because the wavelength of collective 
resonances from metasurface rely on the periodicity of the structure. Here we choose $1 \mu \mathrm{m} \times 1 \mu \mathrm{m}$ unit cell dimensions in order to minimize the influence of the collective resonances in the ENZ region of the ITO.

Time-resolved dynamics measurements. The temporal response of the coupled system was performed with a degenerate optical pump-probe setup. The 1000-Hz, 1240-nm light applied here was generated from an optical parametric amplifier (OPA, TOPAS) which is seeded by a Ti:Sapphire laser system (Spitfire, Spectra-Physics). The output beam was split into two portions as the pump and probe beams. The two beams were set to be nearly parallel $\left(\sim 3^{\circ}\right.$ intersection angle $)$ and illuminating the sample surface normally. The transient transmitted intensity of the probe beam was acquired by a biased InGaAs photodetector (DET10N2, Thorlabs, Inc.). The time delay between the pump and the probe pulses was set by routing the probe beam through a motorized stage with retroreflectors.

\section{ASSOCIATED CONTENT}

\section{Supporting Information}

The Supporting Information is available free of charge at...

(1) Linear transmittance spectra of the strongly coupled systems; (2) Periodicity-dependent transmittance spectra of the strongly coupled system; (3) Intensity-dependent open- and closed-aperture Z-scan traces for the strongly coupled system; (4) Estimation of optical nonlinear coefficients from Z-scan traces; (5) Parameters of the laser for Z-scan measurements; (6) Nonlinear coefficients of the reference samples; (7) Electric field intensity at various wavelengths. 


\title{
AUTHOR INFORMATION
}

\section{Corresponding authors}

\author{
Kuidong Wang: kuidong.wang@unistra.fr
}

Thomas Ebbesen: ebbesen@unistra.fr

\section{Author contributions}

K. W., H.-H. H. and T. W. E. conceived the idea; A.-Y. L. and H.-H. H. designed the nanostructures and performed the numerical simulations. K. W. fabricated the samples, conducted all the optical measurements and the corresponding data analysis; All authors contributed to discussing, interpreting the experimental data, and commenting the manuscript. C. G. and T. W. E. supervised the project.

\section{Funding}

This work of the Interdisciplinary Thematic Institute QMat, as part of the ITI 2021-2028 program of the University of Strasbourg, CNRS and Inserm, was supported by IdEx Unistra (ANR-10IDEX-0002), by SFRI STRAT'US project (ANR-20-SFRI-0012), by the Labex NIE (ANR-11LABX-0058 NIE), CSC (ANR-10-LABX-0026 CSC), the EQUIPEX UNION (ANR-10-EQPX52-01) and PlasHybrid (ANR-18-CE30-0014-02) projects, and by the University of Strasbourg Institute for Advanced Study (USIAS) (ANR-10-IDEX-0002-02) under the framework of the French Investments for the Future Program. The support of the International Center for Frontier Research in Chemistry (icFRC, Strasbourg), the ERC (project no 788482 MOLUSC) and QuantERA (project RouTe) together with the Ministry of Science and Technology of Taiwan 
under the grants MOST 107-2112-M-003-013-MY3 and MOST 110-2112-M-003-020-MY3 are also acknowledged.

\section{Notes}

The authors declare no competing financial interest.

\section{ACKNOWLEDGMENTS}

The authors thank Hicham Majjad for the help of reactive ion etching fabrication, Yifan Yao for the use of the atomic force microscopy and Yuanyuan Zhang for the help of permittivity measurements of ITO with the spectroscopic ellipsometry in East China Normal University. We also thank fruitful discussions with Minghao Li and Gian Lorenzo Paravicini-Bagliani.

\section{REFERENCES}

1. Garcia-Vidal, F. J.; Ciuti, C.; Ebbesen, T. W., Manipulating matter by strong coupling to vacuum fields. Science 2021, 373, eabd0336.

2. Törmä, P.; Barnes, W. L., Strong coupling between surface plasmon polaritons and emitters: a review. Reports on Progress in Physics 2014, 78, 013901.

3. Ebbesen, T. W., Hybrid Light-Matter States in a Molecular and Material Science Perspective. Accounts of Chemical Research 2016, 49, 2403-2412.

4. Christ, A.; Tikhodeev, S. G.; Gippius, N. A.; Kuhl, J.; Giessen, H., Waveguide-Plasmon Polaritons: Strong Coupling of Photonic and Electronic Resonances in a Metallic Photonic Crystal Slab. Physical Review Letters 2003, 91, 183901.

5. Chervy, T.; Xu, J.; Duan, Y.; Wang, C.; Mager, L.; Frerejean, M.; Münninghoff, J. A. W.; Tinnemans, P.; Hutchison, J. A.; Genet, C.; Rowan, A. E.; Rasing, T.; Ebbesen, T. W., High-Efficiency Second-Harmonic Generation from Hybrid Light-Matter States. Nano Letters 2016, 16, 7352-7356. 
6. Chervy, T.; Azzini, S.; Lorchat, E.; Wang, S.; Gorodetski, Y.; Hutchison, J. A.; Berciaud, S.; Ebbesen, T. W.; Genet, C., Room Temperature Chiral Coupling of Valley Excitons with Spin-Momentum Locked Surface Plasmons. ACS Photonics 2018, 5, 1281-1287.

7. Liu, X.; Yi, J.; Li, Q.; Yang, S.; Bao, W.; Ropp, C.; Lan, S.; Wang, Y.; Zhang, X., Nonlinear Optics at Excited States of Exciton Polaritons in Two-Dimensional Atomic Crystals. Nano Letters 2020, 20, 16761685.

8. Barachati, F.; Simon, J.; Getmanenko, Y. A.; Barlow, S.; Marder, S. R.; Kéna-Cohen, S., Tunable Third-Harmonic Generation from Polaritons in the Ultrastrong Coupling Regime. ACS Photonics 2018, 5, 119-125.

9. Liu, B.; Crescimanno, M.; Twieg, R. J.; Singer, K. D., Dispersion of Third-Harmonic Generation in Organic Cavity Polaritons. Advanced Optical Materials 2019, 7, 1801682.

10. Bruno, V.; DeVault, C.; Vezzoli, S.; Kudyshev, Z.; Huq, T.; Mignuzzi, S.; Jacassi, A.; Saha, S.; Shah, Y. D.; Maier, S. A.; Cumming, D. R. S.; Boltasseva, A.; Ferrera, M.; Clerici, M.; Faccio, D.; Sapienza, R.; Shalaev, V. M., Negative Refraction in Time-Varying Strongly Coupled Plasmonic-Antenna--EpsilonNear-Zero Systems. Physical Review Letters 2020, 124, 043902.

11. Xiang, B.; Ribeiro, R. F.; Li, Y.; Dunkelberger, A. D.; Simpkins, B. B.; Yuen-Zhou, J.; Xiong, W., Manipulating optical nonlinearities of molecular polaritons by delocalization. Science Advances 2019, 5, eaax5196.

12. Xiang, B.; Wang, J.; Yang, Z.; Xiong, W., Nonlinear infrared polaritonic interaction between cavities mediated by molecular vibrations at ultrafast time scale. Science Advances 2021, 7, eabf6397.

13. Wang, K.; Seidel, M.; Nagarajan, K.; Chervy, T.; Genet, C.; Ebbesen, T., Large optical nonlinearity enhancement under electronic strong coupling. Nature Communications 2021, 12, 1486.

14. Alam, M. Z.; Schulz, S. A.; Upham, J.; De Leon, I.; Boyd, R. W., Large optical nonlinearity of nanoantennas coupled to an epsilon-near-zero material. Nature Photonics 2018, 12, 79-83.

15. Kuznetsov, A. I.; Miroshnichenko, A. E.; Brongersma, M. L.; Kivshar, Y. S.; Luk'yanchuk, B., Optically resonant dielectric nanostructures. Science 2016, 354, aag2472.

16. West, P. R.; Ishii, S.; Naik, G. V.; Emani, N. K.; Shalaev, V. M.; Boltasseva, A., Searching for better plasmonic materials. Laser \& Photonics Reviews 2010, 4, 795-808.

17. Bakker, R. M.; Permyakov, D.; Yu, Y. F.; Markovich, D.; Paniagua-Domínguez, R.; Gonzaga, L.; Samusev, A.; Kivshar, Y.; Luk'yanchuk, B.; Kuznetsov, A. I., Magnetic and Electric Hotspots with Silicon Nanodimers. Nano Letters 2015, 15, 2137-2142.

18. Liu, S.; Sinclair, M. B.; Saravi, S.; Keeler, G. A.; Yang, Y.; Reno, J.; Peake, G. M.; Setzpfandt, F.; Staude, I.; Pertsch, T.; Brener, I., Resonantly Enhanced Second-Harmonic Generation Using III-V Semiconductor All-Dielectric Metasurfaces. Nano Letters 2016, 16, 5426-5432. 
19. Koshelev, K.; Kruk, S.; Melik-Gaykazyan, E.; Choi, J.-H.; Bogdanov, A.; Park, H.-G.; Kivshar, Y., Subwavelength dielectric resonators for nonlinear nanophotonics. Science 2020, 367, 288.

20. Shcherbakov, M. R.; Neshev, D. N.; Hopkins, B.; Shorokhov, A. S.; Staude, I.; Melik-Gaykazyan, E. V.; Decker, M.; Ezhov, A. A.; Miroshnichenko, A. E.; Brener, I.; Fedyanin, A. A.; Kivshar, Y. S., Enhanced Third-Harmonic Generation in Silicon Nanoparticles Driven by Magnetic Response. Nano Letters 2014, 14, 6488-6492.

21. Yang, Y.; Wang, W.; Boulesbaa, A.; Kravchenko, I. I.; Briggs, D. P.; Puretzky, A.; Geohegan, D.; Valentine, J., Nonlinear Fano-Resonant Dielectric Metasurfaces. Nano Letters 2015, 15, 7388-7393.

22. Liu, S.; Vabishchevich, P. P.; Vaskin, A.; Reno, J. L.; Keeler, G. A.; Sinclair, M. B.; Staude, I.; Brener, I., An all-dielectric metasurface as a broadband optical frequency mixer. Nature Communications 2018, 9, 2507.

23. Liu, H.; Guo, C.; Vampa, G.; Zhang, J. L.; Sarmiento, T.; Xiao, M.; Bucksbaum, P. H.; Vučković, J.; Fan, S.; Reis, D. A., Enhanced high-harmonic generation from an all-dielectric metasurface. Nature Physics 2018, 14, 1006-1010.

24. Shcherbakov, M. R.; Vabishchevich, P. P.; Shorokhov, A. S.; Chong, K. E.; Choi, D.-Y.; Staude, I.; Miroshnichenko, A. E.; Neshev, D. N.; Fedyanin, A. A.; Kivshar, Y. S., Ultrafast All-Optical Switching with Magnetic Resonances in Nonlinear Dielectric Nanostructures. Nano Letters 2015, 15, 6985-6990.

25. Shcherbakov, M. R.; Liu, S.; Zubyuk, V. V.; Vaskin, A.; Vabishchevich, P. P.; Keeler, G.; Pertsch, T.; Dolgova, T. V.; Staude, I.; Brener, I.; Fedyanin, A. A., Ultrafast all-optical tuning of direct-gap semiconductor metasurfaces. Nature Communications 2017, 8, 17.

26. Grinblat, G.; Zhang, H.; Nielsen, M. P.; Krivitsky, L.; Berté, R.; Li, Y.; Tilmann, B.; Cortés, E.; Oulton, R. F.; Kuznetsov, A. I.; Maier, S. A., Efficient ultrafast all-optical modulation in a nonlinear crystalline gallium phosphide nanodisk at the anapole excitation. Science Advances 2020, 6, eabb3123.

27. Reshef, O.; De Leon, I.; Alam, M. Z.; Boyd, R. W., Nonlinear optical effects in epsilon-near-zero media. Nature Reviews Materials 2019, 4, 535-551.

28. Wang, K.; Li, M.; Hsiao, H.-H.; Zhang, F.; Seidel, M.; Liu, A.-Y.; Chen, J.; Devaux, E.; Genet, C.; Ebbesen, T., High Contrast, Femtosecond Light Polarization Manipulation in Epsilon-near-Zero Material Coupled to a Plasmonic Nanoantenna Array. ACS Photonics 2021, 8, 2791-2799.

29. Bohn, J.; Luk, T. S.; Tollerton, C.; Hutchings, S. W.; Brener, I.; Horsley, S.; Barnes, W. L.; Hendry, E., All-optical switching of an epsilon-near-zero plasmon resonance in indium tin oxide. Nature Communications 2021, 12, 1017.

30. Sheik-Bahae, M.; Said, A. A.; Wei, T.; Hagan, D. J.; Stryland, E. W. V., Sensitive measurement of optical nonlinearities using a single beam. IEEE Journal of Quantum Electronics 1990, 26, 760-769. 
31. Vassant, S.; Hugonin, J.-P.; Marquier, F.; Greffet, J.-J., Berreman mode and epsilon near zero mode. Optics Express 2012, 20, 23971-23977.

32. Vassant, S.; Archambault, A.; Marquier, F.; Pardo, F.; Gennser, U.; Cavanna, A.; Pelouard, J. L.; Greffet, J. J., Epsilon-Near-Zero Mode for Active Optoelectronic Devices. Physical Review Letters 2012, 109, 237401.

33. Campione, S.; Brener, I.; Marquier, F., Theory of epsilon-near-zero modes in ultrathin films. Physical Review B 2015, 91, 121408.

34. Jun, Y. C.; Reno, J.; Ribaudo, T.; Shaner, E.; Greffet, J.-J.; Vassant, S.; Marquier, F.; Sinclair, M.; Brener, I., Epsilon-Near-Zero Strong Coupling in Metamaterial-Semiconductor Hybrid Structures. Nano Letters 2013, 13, 5391-5396.

35. Staude, I.; Miroshnichenko, A. E.; Decker, M.; Fofang, N. T.; Liu, S.; Gonzales, E.; Dominguez, J.; Luk, T. S.; Neshev, D. N.; Brener, I.; Kivshar, Y., Tailoring Directional Scattering through Magnetic and Electric Resonances in Subwavelength Silicon Nanodisks. ACS Nano 2013, 7, 7824-7832.

36. Long, S.; McAllister, M.; Liang, S., The resonant cylindrical dielectric cavity antenna. IEEE Transactions on Antennas and Propagation 1983, 31, 406-412.

37. Neira, A. D.; Olivier, N.; Nasir, M. E.; Dickson, W.; Wurtz, G. A.; Zayats, A. V., Eliminating material constraints for nonlinearity with plasmonic metamaterials. Nature Communications 2015, 6, 7757. 38. Alam, M. Z.; De Leon, I.; Boyd, R. W., Large optical nonlinearity of indium tin oxide in its epsilonnear-zero region. Science 2016, 352, 795.

39. Grinblat, G.; Berté, R.; Nielsen, M. P.; Li, Y.; Oulton, R. F.; Maier, S. A., Sub-20 fs All-Optical Switching in a Single Au-Clad Si Nanodisk. Nano Letters 2018, 18, 7896-7900.

40. Li, W.; Chen, B.; Meng, C.; Fang, W.; Xiao, Y.; Li, X.; Hu, Z.; Xu, Y.; Tong, L.; Wang, H.; Liu, W.; Bao, J.; Shen, Y. R., Ultrafast All-Optical Graphene Modulator. Nano Letters 2014, 14, 955-959.

41. Smirnova, D. A.; Khanikaev, A. B.; Smirnov, L. A.; Kivshar, Y. S., Multipolar Third-Harmonic Generation Driven by Optically Induced Magnetic Resonances. ACS Photonics 2016, 3, 1468-1476.

42. Kruk, S. S.; Camacho-Morales, R.; Xu, L.; Rahmani, M.; Smirnova, D. A.; Wang, L.; Tan, H. H.; Jagadish, C.; Neshev, D. N.; Kivshar, Y. S., Nonlinear Optical Magnetism Revealed by Second-Harmonic Generation in Nanoantennas. Nano Letters 2017, 17, 3914-3918.

43. Wang, K.; Chen, L.; Zhang, H.; Hsiao, H.-H.; Tsai, D. P.; Chen, J., Plasmon-enhanced optical nonlinearity for femtosecond all-optical switching. Applied Physics Letters 2017, 111, 181102.

44. Boyd, R. W., Nonlinear optics. Elsevier: 2003.

45. Boyd, R. W.; Shi, Z.; De Leon, I., The third-order nonlinear optical susceptibility of gold. Optics Communications 2014, 326, 74-79. 
46. Caspani, L.; Kaipurath, R. P. M.; Clerici, M.; Ferrera, M.; Roger, T.; Kim, J.; Kinsey, N.; Pietrzyk, M.; Di Falco, A.; Shalaev, V. M.; Boltasseva, A.; Faccio, D., Enhanced Nonlinear Refractive Index in \$lensuremath\{lepsilon\}\$-Near-Zero Materials. Physical Review Letters 2016, 116, 233901.

47. del Coso, R.; Solis, J., Relation between nonlinear refractive index and third-order susceptibility in absorbing media. J. Opt. Soc. Am. B 2004, 21, 640-644.

48. Guo, P.; Schaller, R. D.; Ketterson, J. B.; Chang, R. P., Ultrafast switching of tunable infrared plasmons in indium tin oxide nanorod arrays with large absolute amplitude. Nature Photonics 2016, 10, 267.

49. Kinsey, N.; DeVault, C.; Kim, J.; Ferrera, M.; Shalaev, V. M.; Boltasseva, A., Epsilon-near-zero Al-doped $\mathrm{ZnO}$ for ultrafast switching at telecom wavelengths. Optica 2015, 2, 616-622.

50. Clerici, M.; Kinsey, N.; DeVault, C.; Kim, J.; Carnemolla, E. G.; Caspani, L.; Shaltout, A.; Faccio, D.; Shalaev, V.; Boltasseva, A.; Ferrera, M., Controlling hybrid nonlinearities in transparent conducting oxides via two-colour excitation. Nature Communications 2017, 8, 15829.

51. Secondo, R.; Khurgin, J.; Kinsey, N., Absorptive loss and band non-parabolicity as a physical origin of large nonlinearity in epsilon-near-zero materials. Optical Materials Express 2020, 10, 1545-1560. 52. Wang, H.; Du, K.; Jiang, C.; Yang, Z.; Ren, L.; Zhang, W.; Chua, S. J.; Mei, T., Extended Drude Model for Intraband-Transition-Induced Optical Nonlinearity. Physical Review Applied 2019, 11, 064062. 53. Khurgin, J. B.; Clerici, M.; Kinsey, N., Fast and Slow Nonlinearities in Epsilon-Near-Zero Materials. Laser \& Photonics Reviews 2021, 15, 2000291.

54. Ohara, K.; Yamada, T.; Tahara, H.; Aharen, T.; Hirori, H.; Suzuura, H.; Kanemitsu, Y., Excitonic enhancement of optical nonlinearities in perovskite $\mathrm{CH} 3 \mathrm{NH} 3 \mathrm{PbCl} 3$ single crystals. Physical Review Materials 2019, 3, 111601. 